\title{
RESULTS OF BICYCLE EXERCISE STRESS TEST IN PATIENTS WITH STABLE CORONARY HEART DISEASE DEPENDING ON THE ANGIOGRAPHIC SIGNS OF ATHEROSCLEROTIC LESIONS OF CORONARY ARTERIES
}

\author{
Vira Tseluyko \\ Department of Cardiology and Functional Diagnostics ${ }^{l}$ \\ viratseluyko@ukr.net \\ Tetiana Pylova \\ Department of Cardiology and Functional Diagnostics ${ }^{1}$ \\ pylovatanya@gmail.com \\ ${ }^{1}$ Kharkiv Medical Academy of Post-graduate Education \\ 58 Amosova str., Kharkiv, Ukraine, 61176
}

\begin{abstract}
The aim. To identify features of exercise response in coronary heart disease (CHD) patients depending on coronary artery condition and to identify factors associated with a positive test in patients with no obstructive coronary artery disease (INOCA).

Materials and methods. The study included 105 patients diagnosed with stable coronary artery disease (CAD) who were hospitalized at the City Clinical Hospital No. 8 of the Kharkiv City Council. The criteria for diagnosis of ischemia with no obstructive coronary artery disease (INOCA) were met by 53 patients who formed group I. Group II included 52 patients who were consistently hospitalized in the period from June to December 2020, and had obstructive CAD for more than $50 \%$ according to their coronary angiography (CAG).

Results. According to the results of bicycle exercise stress test, a positive test was significantly more often registered in group II $-n=30(57.7 \%)$ patients compared to group I $-n=19(35.8 \%)$ patients $(p=0.0249)$. The duration of the test in patients of group I was significantly longer than 420 seconds [290-540], compared with group II -300.0 [210.0-540.0] ( $p=0.0352)$. Also, in patients in group II, the maximum volume of the test performed was probably lower than in group I $(p=0.0324)$. When calculating the double product, it was also found that in group I its value was significantly higher compared to group II ( $p=0.0292)$. In group I there was a significantly higher rate of chronotropic reserve (44.0 [26.0-60.0]), compared with group II ( $p=0.0168)$. Elevated total cholesterol (above $5 \mathrm{mmol} / \mathrm{l}$ ) is a statistically significant and independent factor of a positive exercise test in patients with INOCA (OR, 1.98 [0.9992-3.926], $p=0.05)$. A correlation was found between the level of exercise tolerance and smoking in INOCA-patients $(r=-0.388975, p=0.010899)$. Patients who underwent MINOCA also showed reduced tolerance to exercise $(r=-0.3104$, $p=0.042721)$

Conclusions. The sensitivity of bicycle exercise stress test in patients with CAD depends on the presence and severity of atherosclerotic lesions of the coronary arteries (63\% in stenotic atherosclerosis, $36 \%$ in no obstructive coronary arteries. It was found that exercise test duration, double product, chronotropic and inotropic reserve of the heart in patients with a positive exercise test with INOCA were significantly higher compared with patients with obstructive CAD. Independent factors associated with a positive exercise test in patients with no obstructive CAD are an increase in total cholesterol (multivariate regression logistic analysis).

Keywords: ischemia and no obstructive coronary artery disease, INOCA, chronic coronary syndrome, ECG exercise test.
\end{abstract}

DOI: $10.21303 / 2504-5679.2021 .001844$

\section{Introduction}

It is known that bicycle exercise stress test is a generally accepted test for detecting myocardial ischemia, provided that there is a mismatch between myocardial oxygen demand and its delivery [1]. Usually, this test is highly informative in the presence of stenotic atherosclerosis, however, according to the literature, in 10-30\% of patients (with a frequency of $50 \%$ in female and $20 \%$ in male) with chest pain are found to have non-obstructive CAD at coronary angiography [2]. Diagnosis of INOCA is a major diagnostic dilemma in modern practice. The most common causes are microvascular dysfunction or vasospastic angina or a combination thereof $[3,4]$. Coronary 
angiography helps to determine the degree of narrowing of the lumen of the main heart vessels. Invasive cardiac catheterization with intracoronary infusion of endothelium-dependent and endothelium-independent vasodilators is used to confirm or exclude coronary vasospasm [2, 5]. However, such methods have an inherent risk and are limited by the capabilities and provision of catheterization laboratories, the experience of the operator performing the procedure [6, 7].

Despite the significant development of invasive methods for the diagnosis of coronary heart disease and non-invasive methods of myocardial imaging, exercise tests remain one of the most accessible methods of functional diagnosis and examination of patients with angina, risk stratification, assessment of functional status of patients, antianginal therapy efficiency. The level of exercise tolerance (ET) and the degree of reactivity of the cardiovascular system indicate the quality of life of a patient with CHD [8]. Bicycle exercise stress test allows to check the response of blood pressure (BP) and heart rate (HR) to dosed exercise and to determine the level of ET, myocardial oxygen consumption and myocardial reserves. These indicators are important in the overall adaptation of the organism [9]. To date, the indicators of bicycle exercise stress test in patients with INOCA have not been studied enough, which requires further research in this area.

The aim. To identify features of exercise response in coronary heart disease (CHD) patients depending on coronary artery condition and to identify factors associated with a positive test in patients with no obstructive coronary artery disease (INOCA).

\section{Materials and methods}

For preliminary analysis, 1,060 angiographies of patients with stable CHD were enrolled. Included patients were hospitalized in the period from October 2018 to February 2021 at the City Clinical Hospital No. 8 of the Kharkiv City Council, Ukraine. There were 53 (5\%) patients who match the criteria for the INOCA diagnosis and enrolled for further study and formed group I. Group II included $n=52$ patients diagnosed with CHD and according to CAG, had obstructive CA for more than $50 \%$. The coronary heart disease was diagnosed on the basis of classic clinical manifestations of angina [4], reliable data in favour of myocardial ischemia according to Holter ECG monitoring in the form of depression/elevation of ST segment (horizontal or descending decrease of ST segment by $0.1 \mathrm{mV}$ at the point, elevation of the ST segment by $0.1 \mathrm{mV}$ lasting $80 \mathrm{~ms}$ from point $\mathrm{J}$ ), as well as with past history of verified myocardial infarction $[4,10]$.

The work was approved by the Bioethics Committee of Kharkiv Medical Academy of Post-graduate Education (Report No. 8 of $19^{\text {th }}$ October 2018) in accordance with the Code of Ethics of the World Medical Association (Helsinki Declaration). All patients included in study provided written inform consent.

Exclusion criteria were severe comorbidities (valve defects, cancer, chronic kidney disease (CKD) above stage II, severe type 2 diabetes mellitus (DM), etc.), acute coronary syndrome. Clinical and anamnestic characteristics of patients are shown in Table $\mathbf{1 .}$

All patients underwent general clinical and instrumental research methods in accordance with the standards for the diagnosis of coronary heart disease [4]. Pentra Enzymatic ABX reagent was used to determine the level of creatinine by the enzymatic method with colorimetric control. GFR was calculated by the formula CKD-EPI [11]. Indicators of lipid metabolism: the content of total cholesterol (TC), triglycerides (TG), high-density lipoprotein cholesterol (HDL cholesterol) was determined by enzymatic method on a stat analyser Stat Fax 1904 plus (USA).

CAG was performed mainly by right transradial access, the method of selective catheterization of the CA on the appliance TOSHIBA INFX-8000C (Japan) with software according to conventional methods. The presence of hemodynamically significant CA stenosis was assessed according to the ANA classification [12].

All patients underwent bicycle exercise stress test to verify the functional class of stable angina and establish the exercise tolerance. The research was performed on a bicycle ergometer Kettler (Germany), in the morning 1.5-2 hours after a light breakfast, at an air temperature of $18-20{ }^{\circ} \mathrm{C}$ according to Zharinov step-like method $[13,14]$. Two days before the test, $\beta$-blockers were discontinued. The initial load level was $50 \mathrm{~W}$, with each step the load increased by $25 \mathrm{~W}$ every 3 minutes at a speed of 60-80 revolutions per minute until the end points were reached. 
Criteria for a positive bicycle exercise stress test were: the occurrence of typical angina pain, horizontal or oblique depression, or elevation of the ST segment by $1 \mathrm{~mm}$ or more from the isoline lasting about 60-80 ms after the end of the QRS complex. Lack of adequate increase in systolic blood pressure or its decrease with increasing load capacity (with or without ECG signs of myocardial ischemia) during the exercise test. Criteria for cessation were: achieving submaximal heart rate, feeling short of breath, significant dyspnoea, asphyxiation, arrhythmia (ventricular tachycardia, frequent ventricular arrhythmias, supraventricular tachycardia, atrioventricular block of II-III types, bradycardia, His bundle branch block); increase in systolic blood pressure over $230 \mathrm{~mm} \mathrm{Hg}$ and/or diastolic blood pressure over $115 \mathrm{~mm} \mathrm{Hg}$; symptoms of the central nervous system (ataxia, dizziness, presyncope); signs of insufficient peripheral perfusion (cyanosis, pallor, cramps in the lower extremities, transient lameness); significant weakness, fatigue, the patient's request to stop the exercise test. ECG recording was performed at the end of each load step without stopping pedalling. Blood pressure was measured 30 seconds before the end of pedalling the set load power. The patients also had their blood pressure and heart rate measured at rest.

Table 1

Clinical and anamnestic characteristics of the examined groups of patients

\begin{tabular}{lcc}
\hline \multicolumn{1}{c}{ Parameters } & Group I $\boldsymbol{n}=\mathbf{5 3}$ & Group II $\boldsymbol{n}=\mathbf{5 2}$ \\
\hline Age, years $(M \pm \sigma)$ & $56.83 \pm 9.72$ & $60.12 \pm 9.94$ \\
Female, $n(\%)$ & $35 / 66.0$ & $17 / 32.7^{*}$ \\
Male, $n(\%)$ & $18 / 34.0$ & $35 / 67.3^{*}$ \\
AH in the anamnesis, $n(\%)$ & $38 / 71.7$ & $42 / 80.8$ \\
Diabetes mellitus, $n(\%)$ & $8 / 15.1$ & $16 / 30.8$ \\
Hyperlipidemia ${ }^{1}, n(\%)$ & $31 / 58.5$ & $20 / 38.5^{*}$ \\
Smoking, $n(\%)$ & $12 / 22.6$ & $15 / 28.8$ \\
Heredity ${ }^{2}, n(\%)$ & $3 / 5.7$ & $5 / 9.6$ \\
MI in the anamnesis, $n(\%)$ & $16 / 30.2$ & $31 / 59.9^{*}$ \\
CKD in the anamnesis ${ }^{3}, n(\%)$ & $2 / 3.8$ & 0 \\
Stroke, TIA, $n(\%)$ & $3 / 5.7$ & $5 / 9.6$ \\
BMI, kg/m ${ }^{2}$ & $28.26 \pm 4.00$ & $27.81 \pm 3.38$ \\
Typical angina & $23 / 43.4$ & $39 / 75^{*}$ \\
Atypical angina & $30 / 56.6$ & $13 / 25^{*}$
\end{tabular}

Note: ${ }^{*} p<0.05$ comparison between patient with no obstructive CAD and with obstructive CAD. ${ }^{1}$ Hyperlipidemia: total cholesterol $>5.2 \mathrm{mmol} / \mathrm{l}$, and/or low-density lipoprotein cholesterol $>3 \mathrm{mmol} / \mathrm{l}$, and/or triglycerides $>1.7 \mathrm{mmol} / \mathrm{l}$. ${ }^{2}$ Heredity: family history of premature cardiovascular disease (males - under the age of 55; females - under the age of 60). ${ }^{3} C K D$ in the anamnesis: CKD of stage III-IV (reducing glomerular filtration rate accordingly $30-59\left(\mathrm{ml} / \mathrm{min} / 1.73 \mathrm{~m}^{2}\right)$ and $15-29\left(\mathrm{ml} / \mathrm{min} / 1.73 \mathrm{~m}^{2}\right)$. AH - arterial hypertension; TIA - transient ischemic attacks

Chronotropic heart function was determined using chronotropic reserve (HR):

$$
\mathrm{HR}=\text { load heart rate }- \text { resting heart rate. }
$$

The state of inotropic heart function during exercise was calculated by determining the inotropic reserve (IR):

$$
\mathrm{IR}=\text { load SBP }- \text { resting SBP. }
$$

A low increase in IR indicates a decrease in myocardial reserves.

Double product (DP) at the final stage of physical activity was determined by the formula:

$$
\mathrm{DP}=(\text { Heart rate } \times \text { Syst. } \mathrm{BP}) / 100 .
$$


Statistical processing of the obtained data was performed using the statistical software package Statistica 8.0 (StatSoft Inc, USA), Microsoft Office Exel-2003. In the normal distribution, quantitative characteristics were presented as mean \pm standard deviation $(M \pm \sigma)$. Student's test was used to compare the average of the two samples. Intergroup differences in qualitative traits were assessed using Pearson's $\chi^{2}$ test. Multivariate regression logistic analysis was used to determine the indicators that are predictors of a positive exercise test. The coefficient $\beta$, standard error, odds ratio (OR), $95 \%$ confidence interval (CI) for each factor were evaluated. For all types of analysis, the differences were considered statistically significant at $p<0.05$.

\section{Results}

According to the results of bicycle exercise stress test, a positive test with physical activity was significantly more often registered in group II $-n=30(57.7 \%)$ patients compared to group I $-n=19(35.8 \%)$ patients $(p=0.0249)$. A combination of criteria for a positive ECG change test and the occurrence of chest pain $(p=0.0422)$ was statistically significant in patients of group II $n=19$ (36.5 \%) (Fig. 1). Compared with group II, patients in group I were significantly more likely to show changes during restitution, such as the appearance of depression of the ST segment, atrial extrasystoles, and ventricular extrasystoles (Table 2).
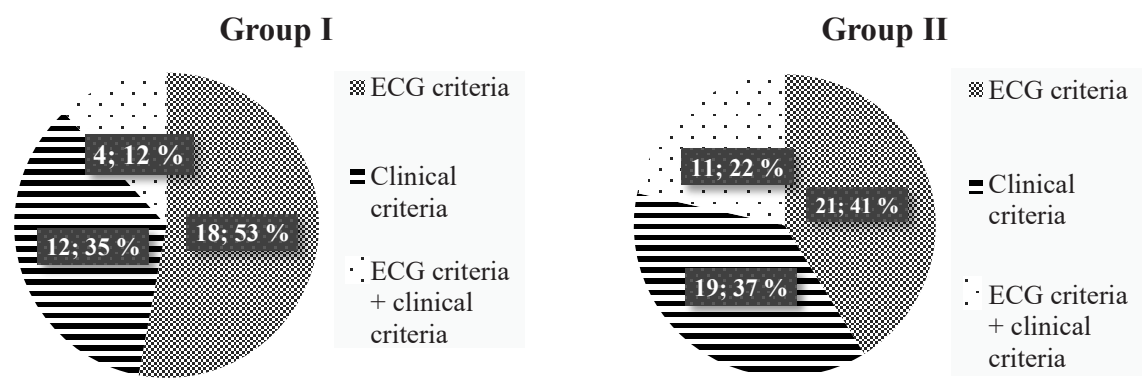

Fig. 1. Criteria for positive bicycle exercise stress test in patients with CAD depending on the condition of the coronary arteries

In patients with INOCA, the sensitivity of bicycle exercise stress test (36 \%) was lower than in patients with stenotic atherosclerosis $(57.7 \%) . n=19$ patients $(36 \%)$ with no obstructive CAD had incomplete test, while Holter ECG monitoring showed signs of ischemia in 39 patients (73.6 \%) in group I and 40 patients ( $77 \%$ ) in group II.

Analysing the exercise tolerance, it was found that in group I the proportion of patients with low tolerance to exercise was $n=14(26.4 \%)$, with medium tolerance $n=13(24.5 \%)$ and with high tolerance $n=11(20.8 \%)$, while in group II $n=19(36.5 \%)$ patients with low tolerance to exercise, $n=14(26.9 \%)$ with medium tolerance, $n=2(3.8 \%)$ with high tolerance were found.

The duration of the test in patients of group I was significantly longer $414.32 \pm 142.10 \mathrm{com}$ pared with group II - 300.0 [210.0-540.0] $(p=0.0352)$. Also, in patients in group II, the maximum volume of the performed test was probably lower than in group I $(p=0.0324)$. When calculating the double product, it was also found that in group I its value was significantly higher compared to group II $(p=0.0292)$.

When determining the chronotropic function of the heart, it was found that group I had a significantly higher rate of chronotropic reserve (44.0 [26.0-60.0]), compared with group II $(p=0.0168)$.

According to the comparative analysis of the features of the response to exercise in patients with stable CHD who had a positive test (Table 3), it was found that in patients of group I the duration of the test was significantly longer than in group II. Patients in group II had significantly lower values of double product, chronotropic and inotropic reserve (respectively $p=0.040$, $p=0.032, p=0.007)$. A high level of exercise tolerance was statistically significantly more often detected in patients in group I, compared with group II $(p=0.0083)$. 
Table 2

The results of bicycle exercise stress test depending on the CA condition in patients with CAD

\begin{tabular}{|c|c|c|}
\hline Parameters & Group I $n=53$ & Group II $n=52$ \\
\hline Positive test, $n(\%)$ & $19 / 35.8$ & $30 / 57.7 *$ \\
\hline Negative test, $n(\%)$ & $7 / 13.2 \%$ & 0 \\
\hline Doubtful test, $n(\%)$ & $3 / 5.7$ & 0 \\
\hline Incomplete test, $n(\%)$ & $19 / 35.8$ & $17 / 32.7$ \\
\hline $\begin{array}{l}\text { Changes in ECG, } n(\%) \text { : } \\
\text { - ST segment depression } \\
\text { - ST segment elevation }\end{array}$ & $\begin{array}{c}15 / 28.3 \\
3 / 5.7\end{array}$ & $\begin{array}{c}21 / 40.4 \\
0\end{array}$ \\
\hline Occurrence of typical angina pain, $n(\%)$ & $12 / 22.6$ & $19 / 36.5$ \\
\hline Combination of ECG criteria + angina pain & $4 / 7.5$ & $11 / 21.2^{*}$ \\
\hline Nonspecific changes in the ECG as a negative $T$ & 0 & $1 / 1.9$ \\
\hline Arrhythmias, $n(\%)$ & $4 / 7.5$ & 9/17.3 \\
\hline Features of the restitution period, $n(\%)$ & $11 / 20.8$ & $1 / 1.9 *$ \\
\hline Test duration, $\sec (M \pm \sigma)$ & $414.32 \pm 142.10$ & $356.51 \pm 180.24^{*}$ \\
\hline Threshold load, W $(M \pm \sigma)$ & $90.18 \pm 32.16$ & $90.12 \pm 21.23$ \\
\hline Maximum load capacity, $(M \pm \sigma)$ & $3046.50 \pm 1691.69$ & $2403.08 \pm 1571.09 *$ \\
\hline Double product, units $(M \pm \sigma)$ & $201.32 \pm 49.76$ & $178.84 \pm 47.90^{*}$ \\
\hline$\Delta$ Double product, units & $113.45 \pm 45.97$ & $90.55 \pm 38.23^{*}$ \\
\hline IR, $\mathrm{mm} \mathrm{Hg}$ & $39.53 \pm 28.76$ & $35.10 \pm 20.81$ \\
\hline $\mathrm{HR}$, beats/min & $40.04 \pm 25.13$ & $30.33 \pm 20.27 *$ \\
\hline Confirmation of ischemia signs on Holter ECG & $39(73.6)$ & 41(77.4) \\
\hline Low tolerance to exercise & $14 / 26.4$ & $19 / 36.5$ \\
\hline Medium tolerance to exercise & $13 / 24.5$ & $14 / 26.9$ \\
\hline High tolerance to exercise & $11 / 20.8$ & $2 / 3.8^{*}$ \\
\hline
\end{tabular}

Note: ${ }^{*}-p<0.05$ comparison between patient with no obstructive CAD and with obstructive CAD

Table 3

Features of positive bicycle exercise stress test depending on the CA condition in patients with CAD

\begin{tabular}{lcc}
\hline \multicolumn{1}{c}{ Parameters } & Group $\mathbf{1} \boldsymbol{n = 1 9}$ & Group $\mathbf{2}=\mathbf{3 1}$ \\
\hline Changes in ECG, $n(\%)$ : & $12 / 63.2$ & 16 \\
- ST segment depression & 0 & 0 \\
- ST segment elevation & $9 / 47.4$ & $19 / 51.6$ \\
Occurrence of typical angina pain, $n(\%)$ & $4 / 21.1$ & $10 / 32.3$ \\
Combination of ECG criteria + angina pain & $2 / 10.5$ & $10 / 32.3$ \\
Arrhythmias, $n(\%)$ & $5 / 26.3$ & $1 / 3.2$ \\
Features of the restitution period, $n(\%)$ & $368.33 \pm 101.99$ & $280.38 \pm 113.44^{*}$ \\
Test duration, sec $(M \pm \sigma)$ & $80.56 \pm 11.02$ & $82.00 \pm 13.54$ \\
Threshold load, W $(M \pm \sigma)$ & $2423.90 \pm 1462.42$ & $1796.40 \pm 825.04$ \\
Maximum load capacity, $(M \pm \sigma)$ & $205.18 \pm 52.66$ & $174.24 \pm 40.30^{*}$ \\
Double product, units $(M \pm \sigma)$ & $106.66 \pm 39.35$ & $82.82 \pm 33.00^{*}$ \\
$\Delta$ Double product, units & $45.26 \pm 18.96$ & $32.90 \pm 19.18^{*}$ \\
IR, mm Hg & $41.47 \pm 19.99$ & $27.16 \pm 17.93^{*}$ \\
HR, beats/min & $8 / 42.1$ & $18 / 58.1$ \\
Low tolerance to exercise & $2 / 10.5$ & $6 / 19.4$ \\
Medium tolerance to exercise & $4 / 21.1$ & $0 * *$
\end{tabular}

Note: ${ }^{*}-p<0.05$ comparison between patient with no obstructive CAD and with obstructive CAD 
To determine the factors associated with a positive exercise test in patients with no obstructive CA, a multivariate regression logistic analysis was performed (Table 4), which proved that elevated total cholesterol (above $5 \mathrm{mmol} / \mathrm{l}$ ) is a statistically significant and independent factor of a positive exercise test in patients with INOCA (OR, 1.98 [0.9992-3.926], $p=0.05$ ). The decrease in GFR-EPI (less than $90 \mathrm{ml} / \mathrm{min}$ ) was close to statistical significance, but no firm significance was obtained (OR, 0.089 [0.7999-1.0098], $p=0.07$ ).

The presence of a correlation between risk factors and the level of exercise tolerance in patients with no obstructive CA was analysed (Table 5).

\section{Table 4}

Multivariate logistic regression analysis of the influence of various factors on a positive exercise test in patients with no obstructive CA

\begin{tabular}{lcccc}
\hline \multicolumn{1}{c}{ Indicators } & $\boldsymbol{\beta}$-coefficient & OR & $\mathbf{9 5} \% \mathbf{C I}$ & $\boldsymbol{p}$ \\
\hline Total cholesterol & Univariate logistic analysis $\left(\chi^{\mathbf{2}=\mathbf{9 . 2 4 2 2}} \mathbf{P}=\mathbf{0 . 0 0 5 1}\right)$ & 0.0503 \\
GFR-EPI & 0.68339 & 1.9806 & 0.9992 up to 3.9260 & 0.0725
\end{tabular}

Table 5

Risk factors and level of exercise tolerance in patients with no obstructive CA

\begin{tabular}{lccc}
\hline \multicolumn{1}{c}{ Parameters } & Quantity $\boldsymbol{N}$ & Spearman's coefficient $\boldsymbol{R}$ & $\boldsymbol{t}(\boldsymbol{N}-\mathbf{2})$ \\
\hline exercise tolerance \& IMT & 43 & -0.259035 & -1.71725 \\
exercise tolerance \& age & 43 & -0.285769 & -1.90944 \\
exercise tolerance \& smoking & 42 & $-0.388975^{*}$ & -2.67039 \\
exercise tolerance \& kidney disease (CKD) & 42 & 0.182482 & 1.17383 \\
exercise tolerance \& hyperlipidaemia & 41 & -0.124839 & -0.78577 \\
exercise tolerance \& heredity & 30 & 0.282321 & 1.55725 \\
exercise tolerance \& hypertensive disease in the anamnesis & 43 & -0.058348 & -0.37424 \\
exercise tolerance \& myocardial infarction in the anamnesis & 43 & $-0.310493^{*}$ & -2.09149 \\
exercise tolerance \& stroke & 42 & 0.182482 & 1.17383 \\
exercise tolerance \& diabetes mellitus & 42 & 0.061372 & 0.38889
\end{tabular}

Note: * - the correlation is statistically significant $p<0.05$

The results of this analysis indicate a negative correlation between the level of exercise tolerance and smoking in patients with INOCA $(p=0.010899)$, there is also a reduced tolerance to exercise in patients undergoing MINOCA $(p=0.042721)$.

\section{Discussion}

When studying the indicators of bicycle exercise stress test, it was found that in patients with no obstructive $\mathrm{CA}$, negative and incomplete exercise test does not exclude the presence of coronary heart disease, which corresponds to the data of other studies [15].

For patients with atherosclerotic lesions of the coronary arteries, the sensitivity of the test was $58 \%$, which corresponds to the data of other researchers, the sensitivity of bicycle exercise stress test is $60-85 \%$, and specificity $-80-88 \%$ [16]. For patients with no obstructive CA, the sensitivity of the test with dosed exercise is slightly lower $-36 \%$, which may indicate that patients with incomplete or negative test are more prone to vasospastic angina, which is confirmed by Holter ECG monitoring [7, 17, 18].

Among patients with a positive test, group I was dominated by patients with clinical criteria of a positive test $-63 \%(n=12)$, which may be due to the peculiarity of cardiac nociception in such patients. It is assumed that severe angina pain is of great importance in the management of patients with no obstructive CAD and requires the appointment of additional anti-ischemic drugs [19]. 
DP reflects myocardial oxygen consumption, load on the cardiovascular system at different times of the day and at different physical exercise. There is a linear relationship between myocardial oxygen consumption and coronary blood flow. At maximum load, coronary blood flow increases 5 times compared to baseline. In obstructive lesions of coronary arteries, the ability to maintain coronary blood flow in the affected area and the maintenance of myocardial metabolic needs during the load is lost, which causes myocardial ischemia [20, 21].

Females (above $66 \%$ were significantly more likely to occur in the group with non-obstructive coronary arteries than in the group of patients with obstructive atherosclerosis $(p=0.006)$. Also, according to the results of multivariate regression analysis, statistically significant factors associated with a positive exercise test in patients with no obstructive CA, were elevated total cholesterol (above $5 \mathrm{mmol} / \mathrm{l}$ ) and decreased GFR (below $90 \mathrm{ml} / \mathrm{min}$ per 1.73 square meters). According to a prospective cohort study WISE, hyperlipidaemia and elevated creatinine are associated with the risk of death in females with non-obstructive CA [13].

Study limitations. Restrictions due to the involvement of small sample of patients.

Prospects for future research. Further studies of non-invasive diagnosis of patients with coronary heart disease with non-obstructive coronary arteries are identified to determine strategies and treatment of patients with INOCA.

\section{Conclusions}

The sensitivity of bicycle exercise stress test in patients with CAD depends on the presence and severity of atherosclerotic lesions of the coronary arteries (with obstructive atherosclerosis $63 \%$, with INOCA - $36 \%$ ).

When comparing data on the positive exercise tests in patients with no obstructive CAD, it was found that the test duration, double product, chronotropic and inotropic reserve of the heart are significantly higher compared with patients with obstructive atherosclerosis. In general, in the group with INOCA, the peculiarity of the response to exercise test was more frequent changes in the ECG during restitution period, such as depression of the ST segment, frequent ventricular arrhythmias.

In patients with no obstructive $\mathrm{CAD}$, a correlation was found between the level of physical exercise (double product, threshold load) and such risk factors as smoking and a history of myocardial infarction.

Independent factors that are associated with a positive exercise test of patients with INOCA are an increase in total cholesterol (multivariate regression logistic analysis).

\section{Conflicts of interests}

The authors declare that they have no conflicts of interest.

\section{Acknowledgments}

We express our gratitude to the patients who took part in the study, as well as the specialists who contributed to the investigation.

\section{References}

[1] Zharinova, O., Ivaniva, Yu., Kutsia, V. (2021). Funktsionalna diahnostyka. Kyiv: Chetverta khvylia, 784.

[2] Bairey Merz, C. N., Pepine, C. J., Walsh, M. N., Fleg, J. L., Camici, P. G., Chilian, W. M. et. al. (2017). Ischemia and No Obstructive Coronary Artery Disease (INOCA). Circulation, 135 (11), 1075-1092. doi: http://doi.org/10.1161/circulationaha.116.024534

[3] Douglas, P. S., Hoffmann, U., Patel, M. R., Mark, D. B., Al-Khalidi, H. R., Cavanaugh, B. et. al. (2015). Outcomes of Anatomical versus Functional Testing for Coronary Artery Disease. New England Journal of Medicine, 372 (14), $1291-1300$. doi: http://doi.org/10.1056/nejmoa1415516

[4] Knuuti, J., Wijns, W., Saraste, A., Capodanno, D., Barbato, E., Funck-Brentano, C. et. al. (2019). 2019 ESC Guidelines for the diagnosis and management of chronic coronary syndromes. European Heart Journal, 41 (3), 407-477. doi: http://doi.org/ 10.1093/eurheartj/ehz425

[5] Fearon, W. F., Kobayashi, Y. (2017). Invasive Assessment of the Coronary Microvasculature. Circulation: Cardiovascular Interventions, 10 (12). doi: http://doi.org/10.1161/circinterventions.117.005361 
[6] Beltrame, J. F., Crea, F., Kaski, J. C., Ogawa, H., Ong, P., Sechtem, U. et. al. (2015). International standardization of diagnostic criteria for vasospastic angina. European Heart Journal, 38 (33), 2565-2568. doi: http://doi.org/10.1093/eurheartj/ehv351

[7] Bairey Merz, C. N. (2019). Testing for Coronary Microvascular Dysfunction. JAMA, 322 (23), 2358. doi: http://doi.org/10.1001/ jama.2019.16625

[8] Rosenmann, D., Mogilevski, Y., Amit, G., Davrath, L. R., Tzivoni, D. (2013). High-frequency QRS analysis improves the specificity of exercise ECG testing in women referred for angiography. Journal of Electrocardiology, 46 (1), $19-26$. doi: http://doi.org/10.1016/j.jelectrocard.2012.08.007

[9] Lanza, G. A., Filice, M., De Vita, A., Villano, A., Manfredonia, L., Lamendola, P., Crea, F. (2018). Microvascular Angina Long-Term Exercise Stress Test Follow-up. Circulation Journal, 82 (4), 1070-1075. doi: http://doi.org/10.1253/circj.cj-17-0657

[10] Kozlov, S. G., Chernova, O. V., Shitov, V. N., Matveeva, M. A., Alekseeva, I. A., Saidova, M. A. (2019). The Diagnostic Accuracy of Exercise Treadmill Testing and Stress Echocardiography for the Detection of Obstructive Coronary Artery Disease in Patients Aged $\geq 70$ Years. Kardiologiia, 59 (10), 23-30. doi: http://doi.org/10.18087/cardio.2019.10.n438

[11] Levey, A. S., Stevens, L. A., Schmid, C. H., Zhang, Y. (Lucy), Castro, A. F. et. al. (2009). A New Equation to Estimate Glomerular Filtration Rate. Annals of Internal Medicine, 150 (9), 604-612. doi: http://doi.org/10.7326/0003-4819-150-9200905050-00006

[12] Neeland, I. J., Patel, R. S., Eshtehardi, P., Dhawan, S., McDaniel, M. C., Rab, S. T. et. al. (2012). Coronary angiographic scoring systems: An evaluation of their equivalence and validity. American Heart Journal, 164 (4), 547-552.e1. doi: http://doi.org/10.1016/j.ahj.2012.07.007

[13] Kenkre, T. S., Malhotra, P., Johnson, B. D., Handberg, E. M., Thompson, D. V., Marroquin, O. C. et. al. (2017). Ten-Year Mortality in the WISE Study (Women's Ischemia Syndrome Evaluation). Circulation: Cardiovascular Quality and Outcomes, 10 (12). doi: http://doi.org/10.1161/circoutcomes.116.003863

[14] Ford, T. J., Yii, E., Sidik, N., Good, R., Rocchiccioli, P., McEntegart, M. et. al. (2019). Ischemia and No Obstructive Coronary Artery Disease. Circulation: Cardiovascular Interventions, 12 (12). doi: http://doi.org/10.1161/circinterventions.119.008126

[15] Nitta, K., Kurisu, S., Sumimoto, Y., Ikenaga, H., Ishibashi, K., Fukuda, Y., Kihara, Y. (2019). Diagnostic value of peak filling rate derived from ECG-gated myocardial perfusion SPECT for detecting myocardial ischaemia in patients with non-obstructive coronary artery disease. Acta Cardiologica, 75 (1), 37-41. doi: http://doi.org/10.1080/00015385.2018.1544698

[16] Sumin, A. N. (2019). Place of clinical evaluation in the identification of obstructive coronary arteries lesions in patients with stable coronary artery disease: Part II. Russian Journal of Cardiology, 8, 111-115. doi: http://doi.org/10.15829/1560-40712019-8-111-115

[17] Goto, M. (2018). Exploring Deep Into the Coronary Microcirculation of Patients With Microvascular Angina. Circulation Journal, 82 (4), 951-953. doi: http://doi.org/10.1253/circj.cj-18-0212

[18] Ong, P., Camici, P. G., Beltrame, J. F., Crea, F., Shimokawa, H., Sechtem, U. et. al. (2018). International standardization of diagnostic criteria for microvascular angina. International Journal of Cardiology, 250, 16-20. doi: http://doi.org/ 10.1016/j.ijcard.2017.08.068

[19] Huang, Q., Wang, J., Li, D., Zhao, J., Feng, X., Zhou, N. (2020). Exercise electrocardiography combined with stress echocardiography for predicting myocardial ischemia in adults. Experimental and Therapeutic Medicine, 21 (2), 130. doi: http://doi.org/10.3892/etm.2020.9562

[20] Beri, N., Dang, P., Bhat, A., Venugopal, S., Amsterdam, E. A. (2019). Usefulness of Excellent Functional Capacity in Men and Women With Ischemic Exercise Electrocardiography to Predict a Negative Stress Imaging Test and Very Low Late Mortality. The American Journal of Cardiology, 124 (5), 661-665. doi: http://doi.org/10.1016/j.amjcard.2019.05.065

[21] Rachwan, R. J., Mshelbwala, F. S., Dardari, Z., Batal, O. (2019). False-positive stress echocardiograms: Predictors and prognostic relevance. International Journal of Cardiology, 296, 157-163. doi: http://doi.org/10.1016/j.ijcard.2019.08.037

How to cite: Tseluyko, V., Pylova, T. (2021). Results of bicycle exercise stress test in patients with stable coronary heart disease depending on the angiographic signs of atherosclerotic lesions of coronary arteries. EUREKA: Health Sciences, 3, 53-60. doi: http://doi.org/10.21303/2504-5679.2021.001844 